\title{
794 LONG-TERM ANTI-TUMOR PRECLINICAL EFFICACY OF AN OPTIMIZED ANTI PD-1/IL-7 BIFUNCTIONAL ANTIBODY SUSTAINING ACTIVATION OF PROGENITOR STEM-LIKE CD8 TILS AND DISARMING TREG SUPPRESSIVE ACTIVITY
}

Aurore morello*, Margaux Seite, Justine Durand, Geraldine Teppaz, Virginie Thepenier, caroline Mary, Isabelle Girault, Emmanuelle Wilhem, Nicolas Poirier. OSE Immunotherapeutics, Nantes, France

Background Despite the PD-(L)1 therapy success, a majority of patients remain resistant. PD-1+IL7R+ progenitors CD8 TILs is a key $\mathrm{T}$-cell subset associated with durable $\mathrm{PD}-(\mathrm{L}) 1$ therapy response. However, this subset may rapidly undergo apoptosis and/or being fully exhausted after PD-(L)1 blockade. Some cytokines have the potential to strengthen PD-(L)1 therapy by promoting $\mathrm{T}$ cell survival, however, their clinical developments are limited by a shortened half-life and systemic toxicity. To redirect immunotherapy to tumor-specific $\mathrm{T}$ cells, expressing PD1, we propose to selectively deliver the pro-survival IL-7 to PD-1+ T cells using a bifunctional anti-PD1/IL-7 mutein antibody. We previously described that the anti-PD1/IL-7v abrogated suppressive activity of human Treg. Here we evaluated its preclinical anti-tumor efficacy and how it promotes the response of PD1+IL7R+ tumor-specific T cells.

Methods Proliferation, IFN- $\gamma$, IL-7R signaling, and NFAT assays were tested to determine the mechanism of this antibody. For the suppressive assay, CD4 Treg and autologous CD8 Teff were co-cultured. In vivo experiments were performed in hPD-1 KI immunocompetent or humanized immunodeficient mice.

Results The anti-PD1/IL-7v antibody design has been optimized with a monovalent approach to enhance its biological activity: (1) preserved PD-1 antagonist activity, (2) improved pSTAT5 IL7R signaling, and (3) enhanced in vivo drug exposure and antitumor efficacy. An IL7 mutein has been designed to improve activity on PD1 $+\mathrm{T}$ cells while sparing PD1neg $\mathrm{T}$ cells.Using a chronic antigen stimulation model, anti-PD1/IL-7v restores the proliferation \& survival of both early and fully exhausted CD8 + or CD4+ T cells. Similarly, anti-PD1/IL-7v, but not anti-PD1 alone, reactivates exhausted TILs isolated from human resected tumors. Gene expression analysis by Nanostring showed increase cytotoxicity, antigen presentation, and chemokines signatures. In vivo, anti-PD1/IL-7v demonstrated high monotherapy efficacy $(90 \%)$ in a PD-1 sensitive orthotopic immunocompetent mouse tumor model as well as in a PD-1 refractory tumor model with $70 \%$ of CR vs $15 \%$ for anti-PD-1 alone. A selective higher expansion of stem-like/ progenitors CD8 TILs was observed after therapy with antiPD1/IL-7v compared to anti-PD1. Memory immune response was demonstrated in $100 \%$ of cured mice after tumor rechallenge in the absence of new treatment in 3 different tumor models. Finally, using two different humanized mouse models implanted with human tumors (A549 or MDA-MB231), we confirmed significant preclinical monotherapy efficacy of the anti-PD-1/IL7v.

Conclusions These data highlight the potential of anti-PD1/IL7 bifunctional drug to overcome immunotherapy resistance and to promote durable anti-tumor efficacy by preferentially reinvigorating $\mathrm{PD}-1+\mathrm{IL} 7 \mathrm{R}+$ stem-like progenitors CD8 $\mathrm{T}$ cells.

http://dx.doi.org/10.1136/jitc-2021-SITC2021.794 